\title{
Los consejos comunales y la gerencia social comunitaria
}

\section{Community councils and community social management}

\section{Universidad Simón Bolívar Universidad, Venezuela}

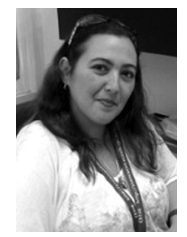

\section{María Fátima Pinho De Oliveira}

Licenciado en Derecho por la Universidad Santa María. Especialista en Derecho Penal. Magister en Derecho Penal. Doctorando en Ciencia Política en la Universidad Simón Bolívar en Caracas. Actualmente es profesora agregada de la Universidad Simón. Ha publicado más de 15 artículos científicos y técnicos en revistas arbitradas y ha participado en congresos nacionales e internacionales. 


\section{Resumen}

La gestión social ha sido definida como la construcción de diversos espacios para la interacción social. Es en estos espacios locales y comunitarios, donde se abren las mayores oportunidades para un acercamiento entre los problemas o necesidades y las comunidades que los padecen y, por consiguiente, representan el ámbito más idóneo para la intervención de la gestión social. El presente trabajo tiene como finalidad dar a conocer los aspectos teóricos sobre cómo actúa la gerencia social comunitaria en favor de los Consejos Comunales.

Palabras clave: Consejo comunal, participación, gestión social, gerencia social y comunitaria.

\section{Abstract}

Social management has been defined as the construction of various spaces for social interaction. It is in these local spaces that opportunities open up between the problems/needs and the communities, and therefore they the spaces - are a more suitable area for social management intervention This paper aims to present the theoretical aspects of how community social management acts in favor of Community Councils.

Keywords: Communal Councils, social management, community management, participation. 


\section{Introducción}

La Constitución de la República Bolivariana de Venezuela [CRBV], de 1999, así como diversos marcos normativos derivados de sus preceptos proponen, sin lugar a dudas, un nuevo modelo de gestión para el ejercicio de la función pública y, por supuesto, una nueva manera de pensar y hacer políticas públicas.

En esta arquitectura jurídica se destaca la participación no sólo como un medio necesario para conferirle eficacia, eficiencia y transparencia a la acción gubernamental, sino además, como un fin en sí mismo otorgándole la categoría de derecho. Podría decirse que el modelo de gestión propuesto por la Constitución es participativo. De ahí que el proceso de formación de políticas públicas debe incluir la participación de actores sociales no gubernamentales, incluyendo, las organizaciones comunitarias y la ciudadanía en general.

Sin duda, son numerosos los artículos constitucionales que aluden a la participación como pilar del sistema democrático y como elemento central de un nuevo modelo de gestión. Se habla de "democracia participativa y protagónica", como también podría hablarse de un modelo de gestión participativo. En el propio Preámbulo Constitucional se destaca el establecimiento de una sociedad participativa y protagónica, dentro de un Estado Federal y descentralizado, donde la soberanía reside en el pueblo y lo público ya no sea exclusivo de los ámbitos de gobierno, ni de sus instancias de decisión.

Se abre así un proceso para la construcción de nuevos espacios, formas y medios de participación popular en las decisiones públicas, que ocupan aproximadamente 130 artículos de la Constitución.

Dentro de un modelo de gestión participativa, se reivindican los ámbitos local y comunitario como espacios en el cual la interacción entre Estado y sociedad civil tiende a ser más efectiva, en base a esto, no se pretende negar la necesidad y posibilidad de la participación en los ámbitos nacional y estatal, pero es necesario reconocer que a medida que se sube de nivel, la participación va asumiendo un carácter más consultivo, que resolutivo y activo.

Es en los espacios locales y comunitarios, donde se abren las mayores oportunidades para un acercamiento entre los problemas o necesidades y las comunidades que los padecen y, por consiguiente, representa el ámbito más idóneo para la intervención de éstas últimas en las diferentes fases del proceso de tomas de decisiones de la gerencia pública. Es en estos ámbitos donde, bajo un modelo de gestión participativa, puede operacionalizarse y organizarse mejor la política pública, con el fin de solucionar problemas que afectan a la comunidad. 


\section{La Gestión Social}

El término gestión social etimológicamente está formado por palabras que provienen del latín. Así, en primer lugar, está el vocablo gestión que emana de gestio que es fruto de la suma de gestus, que significa "hecho", y del sufijo -tio, que puede traducirse como "acción y efecto". En segundo lugar está la palabra social que, a su vez, tiene su origen en el vocablo latino socius que es equivalente a "compañero".

La gestión social ha sido definida como la construcción de diversos espacios para la interacción social. Se trata de un proceso que se lleva a cabo en una comunidad determinada y que se basa en el aprendizaje colectivo, continuo y abierto para el diseño y la ejecución de proyectos que atiendan necesidades y problemas sociales.

La gestión social implica el diálogo entre diversos actores, como los gobernantes, las empresas, las organizaciones civiles y los ciudadanos.

En este sentido, hay que resaltar el hecho de que este tipo de gestión, por las acciones que ella implica y lleva a cabo, trae consigo que esté en relación con otros ámbitos tales como el Derecho, la Educación, el Trabajo Social, la Sociología, la Antropología e incluso la Psicología Social.

La Gestión Social puede definirse según Bernal (2004), como el canal por medio del cual se desarrolla en las personas y en la comunidad un espíritu emprendedor para generar un cambio social, para responder a la búsqueda de la superación de la pobreza e, igualmente para que se adquieran destrezas para abordar un entorno de turbulencia cuyos componentes políticos, económicos, socioculturales, ambientales y tecnológicos o favorecen los procesos de desarrollo sociales; de ahí, que se requiere afianzar los lazos comunitarios, recuperar los valores colectivos y recuperar la identidad cultural, así mismo, implica la convergencia de intereses y necesidades comunes, finalidades humanas concertadas.

\section{Elementos de la Gestión Social}

Conformación y formación de grupos de base: Se basa en la creación de gestores sociales comprometidos con la comunidad. Los gestores sociales, muy especialmente en América Latina, han tomado conciencia sobre la importancia que tiene la formación, sobre todo entre los miembros de los sectores sociales más vulnerables, debido a que éstos han sido intervenidos en múltiples ocasiones y su confianza ha sido diezmada por la cantidad de promesas incumplidas por parte de personas que se acercan a ellos en diferentes épocas. 
El Reconocimiento como personas integrales: El proceso de formación de grupos debe incluir el tiempo necesario para que los integrantes de los mismos puedan reconocerse como personas integrales, como seres humanos que llegan a los grupos con su propia historia, con sus cualidades, defectos, potencialidades, limitaciones, afectos, aficiones, gustos, habilidades, destrezas.

En la fase del diagnóstico es indispensable que los animadores sociales reconozcan que las comunidades y las personas que las integran no sólo son consumidores potenciales. Son actores sociales en permanente movimiento que generan su propia cultura y escriben cotidianamente su propia historia, son dueños de un saber y de unas destrezas que les permiten ser gestores de su propio desarrollo, para lo cual es necesario ayudarles a construir las herramientas para manejar conscientemente su saber y estar en mejores condiciones de afrontar los desafíos. Por lo tanto, la comunidad no es un espacio vacío, carente de conocimientos y de experiencias que pueda ser llenado con los conocimientos de las instituciones y de los profesionales. El Autor Bernal (2004, p.4) refiere que este conocimiento puede lograrse permitiendo a sus integrantes contar quienes son a través de su historia de vida o a través de sus escudos personales y así generar lazos de confianza que le permitan la búsqueda de intereses colectivos.

El diagnóstico: Se basa en el conocimiento previo de la realidad sobre la cual se pretende intervenir. En el diagnóstico se trata de detectar necesidades, expectativas, problemas, fortalezas, debilidades y oportunidades de los integrantes de una determinada comunidad. Este es un punto clave del proceso formativo de los grupos y del esfuerzo por animar la vida asociativa de las personas; se trata de tomar conciencia de la situación de la realidad, de que todos los miembros de la comunidad sepan que pasa porque en la medida que se tenga un conocimiento claro de los problemas será más expedita la búsqueda de soluciones apropiadas.

Las comunidades tienden a organizarse con el objeto de resolver sus necesidades y, de esta manera, mejorar sus condiciones de vida. La posibilidad de organizarse se manifiesta en el momento en que se presentan necesidades o aspiraciones que no han sido satisfechas y que no tienen una solución a corto o mediano plazo a partir de la simple acción individual. La organización surge como producto de la unión de los esfuerzos individuales en la perspectiva de enfrentar de manera conjunta la solución a los problemas detectados y/o promover intereses específicos.

La Participación Comunitaria: Al acercarnos a una definición de la participación comunitaria podemos afirmar que es una postura social de construcción colectiva de propuestas de desarrollo que se fundamentan en las 
necesidades de las poblaciones. Es un proceso de identificación de necesidades y de formulación de alternativas que cuenta con la participación de los actores sociales que se articulan en torno a la gestión del desarrollo.

La participación implica procesos de organización, planeación, liderazgo, de preparación y evaluación de proyectos y de comunicación. Bernal (2004, p.9) define a la participación comunitaria como un proceso de identificación de necesidades y de formulación de alternativas que cuentan con la participación de los actores sociales que se articulan en torno a la gestión del desarrollo.

Las circunstancias sociales, económicas, políticas y culturales que hoy afronta la sociedad colombiana requiere la construcción de comunidades participantes de su desarrollo con visión de futuro, con cultura política y con capacidad para ejercer la gerencia social.

Las comunidades excluidas socialmente en América Latina están llamadas a afrontar el reto de asumir la tarea liberadora de su propio desarrollo para poner en primer plano la necesidad de la inversión social de los gobiernos locales, de los gobiernos regionales, de los gobiernos nacionales, de los organismos mundiales y de las organizaciones no gubernamentales, para construir una sociedad organizada que interactúe con el Estado y con otras entidades, a través de la consolidación de redes de actuación social y política de las comunidades.

La Planeación Estratégica: Es el proceso concertado que permite a los miembros de una comunidad u organización la tarea de enfrentar el futuro. Brinda la oportunidad de identificar un horizonte temporal proyectado a largo plazo, un enfoque global que incluye la comunidad o la organización como totalidad integrada por recursos, capacidad y potencialidad y en especial, que se requiere tomar decisiones basados en juicios y no solo en datos. Contiene los planes de aplicación en toda la organización y establece los objetivos generales de la organización, y busca posicionar a la organización en su entorno. Se precisa y analiza información interna y externa con el fin de evaluar la situación presente de la organización así como su nivel de competitividad con el propósito de anticipar y decidir sobre el direccionamiento de la institución hacia el futuro.

El trabajo en Equipo: Las organizaciones de hoy para ser exitosas tienen que conformar equipos de trabajo, máxime cuando se trata de organizaciones comunitarias donde la sumatoria de recursos es fundamental para obtener los resultados deseados.

Formar equipos de trabajo es provechoso porque permiten:

- Reunir diversos conocimientos y habilidades

- Fomentar la cooperación entre diferentes personas, entre 
diferentes departamentos de una misma organización o entre diferentes organizaciones.

- Participar organizadamente en la elaboración de planes, programas y proyectos.

El Liderazgo: En los procesos de organización y participación comunitaria es preciso reconocer el papel fundamental que juega la existencia de líderes, o sea, de personas que sobresalen en la comunidad por su espíritu de colaboración, su sentido de pertenencia y sobre todo porque estimulan e incentivan a los miembros de sus comunidades a actuar, a decidir, a participar.

Las personas dinámicas, que ejercen influencia en los grupos, que perciben las necesidades, que visualizan soluciones, que tienen capacidad de dirección y control, a quienes todos siguen, son los líderes que se convierten en voceros de los grupos, en catalizadores de sus energías y en promotores de su desarrollo. Los líderes son personas que captan los pensamientos y los sentimientos de sus compañeros y pueden descubrir diferentes alternativas para ayudar a satisfacer las aspiraciones de los grupos a los cuales pertenecen.

Los líderes son el resultado de las circunstancias sobre las cuales los grupos de personas se integran y organizan para alcanzar objetivos comunes. Los grupos perciben las habilidades que deben reunir los líderes de acuerdo con las necesidades, los objetivos y los resultados esperados. De ahí, que los líderes dentro de un grupo pueden variar según el cambio de las circunstancias.

Los líderes se destacan cuando ayudan a sus grupos o comunidades a lograr sus metas u objetivos, también cuando impulsan a sus seguidores a participar activamente en la toma de decisiones.

\section{La Participación}

Los análisis realizados por diversos grupos de la sociedad, encuentran los siguientes rasgos característicos de la participación en el texto constitucional: En la Constitución (1999), la noción de representación queda disminuida y se enfatiza la democracia participativa o directa, al contemplarse un conjunto de mecanismos de ejercicio directo de participación política antes inexistentes en el marco jurídico venezolano.

De acuerdo a esto, se salda el vacío existente en anteriores constituciones que tenían un carácter más representativo que participativo y que, contribuyeron a agudizar la crisis de legitimidad del sistema democrático, poniendo en evidencia la necesidad de “... introducir mecanismos no sólo más 
representativos, sino verdaderos mecanismos de participación, que permitan al ciudadano ejercer la soberanía popular". (Ayala, s/f)

Tiene un carácter principista en tanto se la concibe como elemento constitutivo fundamental de la nación y como un derecho inalienable. Así, el Preámbulo de la CRBV señala que se busca: "...establecer una sociedad democrática, participativa y protagónica...". Mientras que el artículo 62 establece explícitamente que: "Todos los ciudadanos y ciudadanas tienen el derecho de participar libremente en los asuntos públicos, directamente o por medio de sus representantes elegidos o elegidas." Este carácter principista se esboza a lo largo de toda la Constitución al establecerse que la garantía de la mayoría de los derechos contemplados, se deberá hacer por la vía participativa.

El concepto de protagonismo se incorpora de forma explícita lo que pudiera interpretarse en un sentido que pretende garantizar una participación activa de la población. Los mecanismos de participación contemplados en la CRBV, comprenden una amplia gama de posibilidades, como lo establece el artículo 70:

Son medios de participación y protagonismo del pueblo en ejercicio de su soberanía, en lo político: la elección de cargos públicos, el referendo, la consulta popular, la revocatoria del mandato, las iniciativas legislativa, constitucional y constituyente, el cabildo abierto y la asamblea de ciudadanos y ciudadanas cuyas decisiones serán de carácter vinculante, entre otros; y en lo social y económico, las instancias de atención ciudadana, la autogestión, la cogestión, las cooperativas en todas sus formas incluyendo las de carácter financiero, las cajas de ahorro, la empresa comunitaria y demás formas asociativas guiadas por los valores de la mutua cooperación y la solidaridad

\section{La Gerencia Social Comunitaria}

De conformidad con el planteamiento anterior sobre la gestión social se puede llegar entonces a explicar el concepto de gerencia comunitaria, que se refiere a la coordinación colectiva en el manejo de los recursos autogestionados, a través de un pensamiento y proceso estratégico que formule la dirección que guiará la comunidad por medio de la planificación, organización, dirección y control y que busque el cumplimiento de la misión y objetivos a través de estrategias claras, definidas por la propia comunidad. (Pérez, 2012 p.69)

El ser humano es un ser social por naturaleza, que busca estar en equilibrio con su entorno y relacionarse de manera constante con individuos semejantes. Es por esto que han surgido numerosas ciencias dedicadas 
exclusivamente al estudio del hombre en sociedad, a fin de determinar los patrones de conducta y las normas sociales que deben regirse para mantener la paz fundamental en la vida cotidiana (Briceño y otros, 2012).

Con el paso de los años ha cobrado una gran importancia la voz y el derecho del pueblo en la toma de decisiones a nivel gubernamental, estableciéndose de este modo una democracia representativa y participativa en muchos países de América Latina. Sin embargo, las comunidades han desarrollado métodos de gerencia que permiten resolver problemas específicos de manera efectiva y relativamente rápida (Briceño y otros, 2012).

Esto constituye un avance importante para la sociedad, ya que gracias a la gerencia social comunitaria los problemas de un determinado sector o una región pueden ser resueltos tomando en cuenta las necesidades de todas las partes involucradas, obteniendo así beneficios para el pueblo y por el pueblo (Briceño y otros, 2012).

Por lo tanto la Gerencia Social es una estrategia que se base en los criterios de la equidad, la eficacia, la eficiencia y la sostenibilidad, que permite orientar la toma de decisiones, las acciones a seguir y el seguimiento y evaluación de los resultados alcanzados

Es un concepto en constante evolución. Sin trascender las diferencias de base debe compararse con la gerencia privada convencional pero con un elemento clave: la construcción de valor público. La Gerencia Social trasciende la rentabilidad económica y procurar la rentabilidad social. Hoy, en la problemática existente, la gerencia social no debe ser solamente una preocupación por la distribución equitativa del ingreso, sino también por la distribución equitativa del conocimiento.

La gerencia social como enfoque presenta una fusión de los distintos saberes y de los aprendizajes desde las mismas experiencias, de este modo produce una mirada propia sobre los problemas.

La gerencia social es imprescindible para avanzar y mantener un desarrollo sostenido con fuertes cimientos en la realidad histórica de nuestros días. Solo así podremos comprender su importancia en algunas dimensiones contextuales en plano cultural, político y social que deben entenderse para ajustar nuestro estilo de gerencia, entre el Estado y la Sociedad. A la necesidad de reflexionar sobre los nuevos retos que implica la gerencia social en el proceso político de transformación que estamos viviendo, dentro de la nueva institucionalidad venezolana.

La gerencia social, entonces es la activación de mecanismos de operación y prestación de servicios a la ciudadanía, permitiendo la participación 
activa de la comunidad organizada. Y teniendo como premisa todos los valores superiores como la vida, la libertad, la justicia, la solidaridad, la igualdad, la democracia, la ética, la responsabilidad social y los derechos humanos.

En lo referente a la gerencia social comunitaria, es importante destacar que a partir de la primera mitad de la década de los 80, el concepto de capital social fue muy discutido dentro del debate académico y en las discusiones de muchos actores, este concepto se propagó dentro de programas de lucha contra la pobreza y en las técnicas de gestión empresarial; aquí la función del gerente social es la de contribuir al logro de una relación íntegra entre el capital social grupal y el capital social comunitario con un espacio local donde se desarrollará ese capital social, los insumos para lograr los objetivos y los beneficios que serán aportados (Coronado, 2008).

Se entiende por capital social a la capacidad que tiene un grupo humano de emprender acciones colectivas que redunden en beneficio de todos los sus miembros (Lorenzelli, 2003, p.1); el mismo autor lo define como la capacidad que pueden desarrollar los grupos humanos de emprender acciones colectivas que redunden en un beneficio mutuo. (p.3)

Esta capacidad radica tanto en la institucionalidad que rige la vida grupal o comunitaria (leyes, procedimientos, medios de comunicación, forma de resolver conflictos, controles y sanciones), como en las normas y hábitos compartidos.

En virtud de lo anterior y de la exploración de las posibilidades se observa que es viable pasar del capital social individual al capital social comunitario. Esta posibilidad no debería hacer pensar que esta construcción resulta una tarea fácil, muy por el contrario la acumulación del capital social supone una labor permanente y sostenible.

La generación del capital social comunitario supone acciones al interior de grupos compuestos por individuos unidos por lazos de proximidad, lo que se ha mencionado como nivel micro. Por otro lado el capital social comunitario exige dar el paso más allá de los grupos de referencia y generar condiciones que favorezcan a toda la comunidad.

Por lo tanto se reconoce al espacio intermedio o comunitario como el radio de acción privilegiado para la acción de la gerencia social y las políticas públicas en la tarea de generar capital social ya que es a éste nivel donde tienen su máxima expresión los insumos o recursos del capital social. Esto es: la historia en común, los lazos de familia y vecindad así como elementos identitarios que posibilitan una cultura de confianza entre los individuos; estos insumos, de carácter emocional, se complementan con un marco de funcionamiento 
que posibilitan las relaciones entre los individuos o recurso estructural de la comunidad (Lorenzelli, 2003, p.10).

La gerencia social comunitaria nace como una necesidad de administrar los recursos que pertenecen a la sociedad. Este nuevo modelo de administrar, se está utilizando principalmente en los consejos comunales, ya que ellos son los encargados de asignar y dar buen uso a los recursos que les otorga el Estado. Para lograr este objetivo es necesario tomar en cuenta la Ley que rige los Consejos Comunales (Briceño y otros, 2012)

La gerencia comunitaria es una estrategia que se basa en los criterios de equidad, eficacia, la eficiencia y la sostenibilidad, que permite orientar la toma de decisiones, haciendo seguimiento y evaluación de los resultados alcanzados. La gerencia comunitaria es un nuevo enfoque administrativo social que surge como una necesidad de las sociedades que tratan a través de éste modelo involucrar a la comunidad, en cualquiera de sus formas, para que sean parte de la transformación que las mismas viven, debido a los factores económicos que rigen un mundo ya globalizado.

Dentro de este orden de ideas, la gerencia comunitaria trata del empoderamiento de las comunidades, es decir, del acercamiento de los procesos gerenciales y hacer de ellos más eficiente la utilización de los recursos humanos como materiales, para que los proyectos sociales sean ejecutados por la misma comunidad, pero para que esto suceda es necesario la participación activa de todos los actores sociales que la conforman.

\section{Los Consejos Comunales}

Según la Ley de los Consejos Comunales (2006), el Consejo Comunal es un espacio propicio para que las comunidades organizadas y grupos sociales puedan ejercer el derecho a la participación protagónica (artículo $2^{\circ}$ ) consagrado en la Constitución de la República Bolivariana de Venezuela (1999), influyendo en las decisiones que determinan la formación de políticas públicas y la formulación de proyectos en función de la satisfacción de necesidades y aspiraciones de la comunidad. El Consejo comunal ha sido concebido como la agrupación de un número de familias que varía entre 200 y 400, dependiendo si se trata de un área rural o urbana y ha sido asociada a un área geográfica específica, definida por la Ley como "área geográfica de la comunidad" (Ley de los Consejos Comunales, 2006, articulo 4 numeral 6) su delimitación debe ser aprobada en el seno de la Asamblea de Ciudadanos (AC) (León y Martucci, 2009).

El espacio de participación que representa el Consejo Comunal, si 
bien está estrechamente vinculado al gobierno municipal, no mantiene una relación directa con este, ya que se encuentra subordinado al Gobierno nacional por intermedio de la Comisión Nacional Presidencial del Poder Popular y sus instancias regionales y locales, de conformidad con lo dispuesto en la ley (León y Martucci, 2009).

El Consejo Comunal es un medio a través del cual es posible el ejercicio y desarrollo de la ciudadanía, expresada en la dimensión que tiene lugar "entre instituciones o sectores del Estado y colectivos sociales o culturales vinculados a territorios determinados", por lo tanto, en su seno se desarrollaran "procesos conflictivos, de confrontación y de diálogo social que idealmente al final llevan a una nueva formalización política o jurídica", como señala Escobar (2004, p.101), citando a Borja (León y Martucci, 2009).

El contexto en que se desarrollan y fortalecen los Consejos Comunales tiene un horizonte temporal que comienza en 1999 con la aprobación de la Constitución de la República Bolivariana de Venezuela y la transición de la democracia representativa a la participativa. Posteriormente, en 2006 se promulgo la Ley de los Consejos Comunales, instrumento legal que determina su fortalecimiento y reposicionamiento, quedando atrás la relación de dependencia con los Consejos Locales de Planificación que se había establecido en la ley de creación de estos últimos (León y Martucci, 2009).

\section{Reflexiones finales}

Los Consejos Comunales han sido conformados por los respectivos vecinos de la comunidad, cuyos integrantes son seleccionados en el seno comunitario, quedando a discrecionalidad del elector la apreciación la capacidad administrativa y gerencial del elegido, con el agravante de que los integrantes deben tomar decisiones que no solo dependerán de cuestiones racionales sino también en la percepción en que influyen los factores subjetivos, culturales, donde está inserto el libre ejercicio de un poder.

En presencia del capital social los individuos pueden lograr objetivos, individuales y colectivos, que serían más difíciles de materializar si dicho capital no existiera en dosis considerables. Pero es en la combinación con otros tipos de capital que el capital social adquiere su mayor potencialidad, en cambio aislado no se percibe como un elemento importante.

Un grupo de individuos con fuerte cohesión grupal y sentido de pertenencia tienen por ende un acervo de capital social importante. Es el tipo de capital social producto de lazos familiares, amistosos o de alguna otra 
característica que los identifica como similares o pares. Pero este tipo de capital social resulta poco potenciador si el grupo en cuestión está aislado o no tiene contactos con los centros de toma de decisión económica y social. Por lo tanto cobra importancia la capacidad de establecer puentes entre grupos, más allá de los individuos, y eslabonar a los grupos locales con otros ubicados fuera de la comunidad de referencia.

María Fátima Pinho De Oliveira Universidad Simón Bolívar Universidad, Venezuela email: mpinho@usb.ve

Recibido: 23 de noviembre de 2015 Aceptado: 15 de enero de 2016

\section{Referencias}

Bernal, J. (2004) Modulo de Gestión Social. Trabajo de Grado no publicado.

Briceño, J., Mendoza, N., Briceño, R., Becerra,Y., Colmenarez, Z., Principal, Z. (2012). Gerencia Social Comunitaria. UPEL- Fundación Escuela de Gerencia Social. Disponible en: http:// gerenciasocialupel2012.blogspot.com/2012/05/gerencia-social-comunitaria.html. Consulta: 19-11-2015.

Coronado, Yoleida (2008). Gerencia Social Comunitaria: prospectiva o utopía. Revista GERENTIA. Numero 13. Universidad Fermín Toro. Venezuela.

Escobar, A. (2004). Participación ciudadana y políticas publicas. Una problematización acerca de la relación Estado y Sociedad Civil en América Latina en la última década. Revista Austral de Ciencias Sociales (8) 97-108. Recuperado el 14 de mayo de 2007, de http://www. humanidades.uach.cl/ revistas/cssociales_8/n8_articulo7.pdf

León, E., Martucci, A. (2009). Los Consejos Comunales y su articulación con la gerencia pública municipal. Revista Anales. 9 (2) 137-156. Universidad Metropolitana.

Lorenzelli, M. (2003) Capital social comunitario y gerencia social. VIII Congreso Internacional del CLAD sobre la Reforma del Estado y de la Administración Pública, Panamá, 28-31 Oct. 2003

Pérez, J. (2012). Modelo de Gerencia Estratégica para la consolidación de los Consejos Comunales. Trabajo de Grado presentado para optar al título de Magíster en Ciencias Administrativas. Universidad de Oriente. 